\title{
VIBRAÇÃO INDUZIDA POR VÓRTICES: ANÁLISE CRÍTICA DE MODELOS FENOMENOLÓGICOS
}

\author{
VORTEX INDUCED VIBRATIONS: \\ A CRITICAL ANALYSIS OF PHENOMELOGICAL MODELS
}

\author{
Leandro D. Cunha ${ }^{1}$, Celso P. Pesce ${ }^{2}$ \\ Departamento de Engenharia Mecânica da EPUSP \\ ${ }^{1}$ leandro.cunha@poli.usp.br \\ 2 ceppesce@usp.br
}

\begin{abstract}
The present work develops a critical analysis of a family of results emerging from the systematic study of phenomenological models in Vortex Induced Vibration, VIV. The study covers the theoretical foundation, numerical simulation and the applicability of several fenomenological models already available in the literature. Amongst the models presented here are those from Hartlen\&Currie, 1970, Iwan\&Blevins, 1975, and Facchinetti\&de Langre\&Biolley, 2004. The purposes are threefold: based in theoretical and experimental results in the literature, to express the ability of those models in representing de VIV phenomena; to obtain numerical simulations results in order to compare them with experimental data; to address the practical application of the studied models in real engineering cases. The overall conclusion is that the phenomenological models analysed are strongly limited and do not represent the VIV characteristics well enough. An exception is made to the Iwan\&Blevins model for its capacity to predict the peak vibration amplitude.
\end{abstract}

\section{INTRODUÇÃO}

O presente trabalho analisa criticamente um conjunto de resultados sobre a fundamentação, simulação e aplicabilidade de alguns modelos fenomenológicos para Vibração Induzida por Vórtices, ou VIV. Seu conteúdo é baseado na dissertação de Mestrado de Cunha [2]. São estudados os modelos de Hartlen\&Currie [7], 1970, de Iwan\&Blevins [8], 1975, e de Facchinetti\&de Langre\&Biolley [3], 2004.

O estudo foi desenvolvido com os propósitos: fundamentar, com base em resultados teóricos e experimentais existente na literatura, a representatividade do fenômeno de VIV através desta classe de modelos; obter resultados da simulação numérica destes modelos para posterior confrontação com dados experimentais disponíveis na literatura; discutir a possibilidade de aplicação destes modelos na prática da engenharia.

A fundamentação dos modelos fenomenológicos é obtida do trabalho de Aranha [1], 2004, que conclui pela possibilidade teórica de representar os fenômenos de VIV através da equação de Ginzburg-Landau, que pertence à mesma classe da equação de van der Pol, base dos modelos fenomenológicos em VIV. Este fato traz embasamento a tais modelos.

Para desenvolver a simulação numérica dos três modelos foi idealizado um programa de computação científica capaz de resolver numericamente as equações diferenciais ordinárias dos modelos, interpolar os resultados da simulação, efetuar a transformada de Fourier dos resuldados interpolados e apresentar, em um documento formato PDF, um relatório detalhado da simulação. Este relatório permite a análise direta dos dados, sem necessidade de pós-processamento, contendo gráficos de séries temporais, espectro de frequência e diversos gráficos usualmente encontrados na literatura especializada de VIV.

Por sua vez, para discutir a aplicabilidade dos modelos estudados é feita uma compilação da fenomenologia de VIV e os resultados experimentais são comparados com os resultados da simulação. 


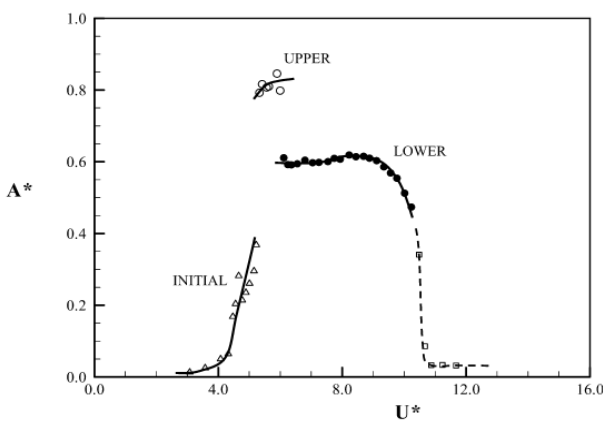

(a) Amplitude de resposta típica de VIV com baixo valor de $m^{*} \zeta$

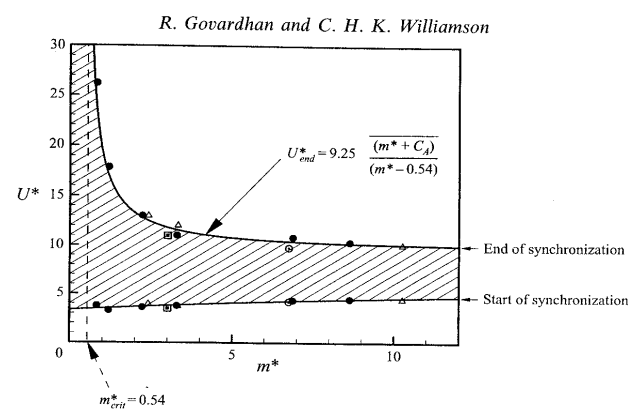

(c) Extensão do domínio de sincronização em função de $m^{*}$

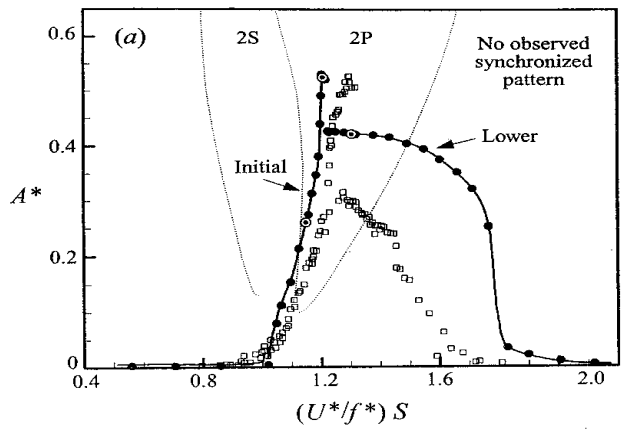

(b) Amplitude de resposta típica de VIV com alto valor de $m^{*} \zeta$

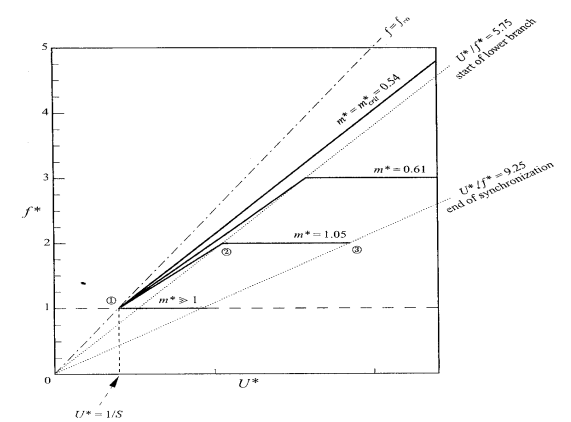

(d) Comportamento da frequência efetiva de vibração

Figura 1: Características do fenômeno de VIV. Fonte [14] e [6]

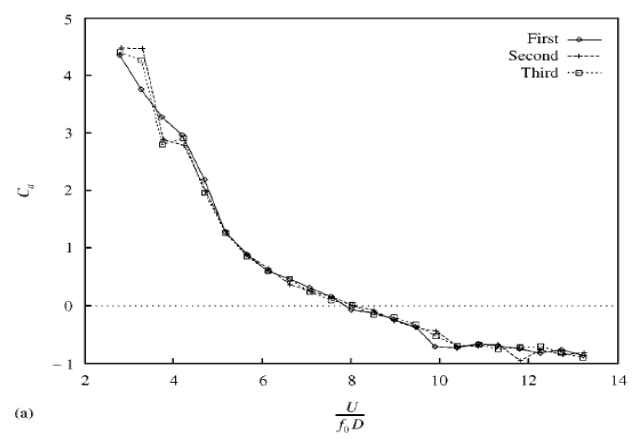

(a) Dados de Vikestad et al. [12]

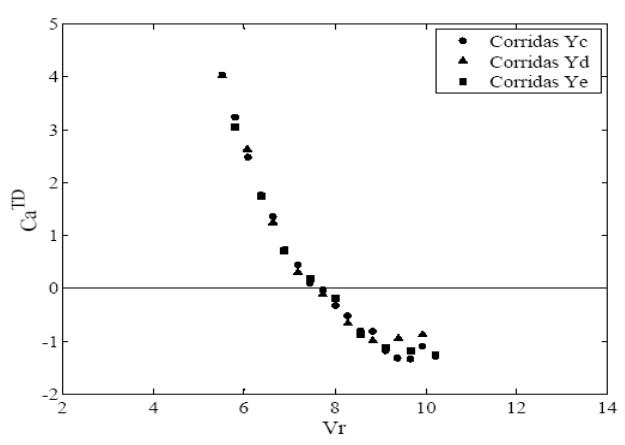

(b) Dados de Fujarra [5]

Figura 2: Dados Experimentais sobre Coeficiente de Massa Adicional

\section{Alguns Aspectos Experimentais do Fenômeno de VIV}

Uma descrição bastante detalhada do fenômeno pode ser encontrada em [10], [11], [14] e, resumidamente, em [2].

Os gráficos das figuras 1a e 1b mostram a amplitude de vibração típica de um cilindro rígido sujeito à VIV. Na figura 1a, nota-se a presença de três ramos distintos na resposta. Para velocidades de escoamento mais baixas tem-se o ramo inicial; próximo à velocidade de ressonância tem-se o ramo superior; e para velocidades elevadas tem-se o ramo inferior. 
Este comportamento caracteriza sistemas com um baixo valor de coeficiente de massaamortecimento, $m^{*} \zeta$, o que é típico de VIV em água.

Para valores mais elevados de $m^{*} \zeta$, figura 1b,típicos de VIV em ar, inexiste diferenciação entre os ramos inicial e superior. Neste caso, o ramo resultante é chamado ramo inicial.

A figura 1c mostra como o dominio de de sincronização se estende conforme se diminui o valor de $m^{*}$. Govardhan\&Williamson [6], baseados em resultados experimentais, deduziram uma expressão analítica para o valor da velocidade em que a sincronização termina. Desta expressão conclui-se que para valores de razão de massa, $m^{*}<0.52$, as vibrações são perenes, ou seja, persistem para velocidades de escoamento bastante elevadas. Esta proposição foi testada experimentalmente e confirmada [6] e [13]. Ainda se desconhece o valor de velocidade de escoamento para o qual as vibrações cessam neste caso.

Nas transições entre os ramos de resposta acontece histerese e resposta intermitente o que caracteriza o fenômeno de VIV como não-linear.

A extensa análise empreendida por Govardhan\&Williamson em [6] permitiu que fosse obtida uma relação entre a razão de massa e a frequência de vibração efetiva do sistema, como mostra a figura 1d. Esta relação é corroborada por uma série de experimentos realizados pelos autores.

A massa adicional do sistema de VIV é um parâmetro representativo tanto da frequência de oscilação quanto da fase relativa entre a força de sustentação e o deslocamento do cilindro. A figura 2 mostra dados experimentais relativos ao cálculo do coeficiente de massa adicional.

\section{Modelos Fenomenológicos em VIV}

Os modelos fenomenológicos estudados foram normalizados para facilitar a identificação e diferenciação de seus parâmetros. Em particular, as equações diferenciais não-lineares foram colocadas na forma da equação de van der Pol.

As equações resultantes para cada modelo são apresentadas abaixo.

A tabela 1 apresenta uma comparação entre os três modelos analisados e o modelo fenomenológico padrão. Cada parâmetro do modelo padrão é identificado com seu correspondente nos três modelos em estudo.

\section{Modelo Fenomenológico Padrão}

$$
\begin{aligned}
& \ddot{y}+2 \zeta_{w} \delta_{w} \dot{y}+\delta_{w}^{2} y=s-\Delta_{m_{a}} \ddot{y}-2 \Delta_{\zeta_{a}} \delta_{w} \dot{y} \\
& \ddot{v}+\epsilon\left(\mu v^{2}-1\right) \dot{v}+v=f \\
& \ddot{y}+2 \zeta_{w} \delta_{w} \dot{y}+\delta_{w}^{2} y=\frac{1}{2 \pi^{3} S t^{2}\left(m^{*}+1\right)} \int_{t_{0}}^{t} v d t+\frac{1}{m^{*}+1} \ddot{y} \\
& \ddot{v}+\alpha\left(3 \frac{\gamma}{\alpha} v^{2}-1\right) \dot{v}+v=b \delta_{n} \ddot{y} \\
& \ddot{y}+2 \zeta_{w} \delta_{w} \dot{y}+\delta_{w}^{2} y=\frac{1}{2 \pi^{3} S t^{2}\left(m^{*}+1\right)} \frac{\alpha_{4}}{\sqrt{\alpha_{2}}}\left(v-2 \pi S t \sqrt{\alpha_{2}} \dot{y}\right)+\frac{1}{m^{*}+1} \ddot{y} \\
& \ddot{v}+2 \alpha_{1}\left(4 v^{2}-1\right) \dot{v}+v=\frac{2}{\pi} \frac{\alpha_{4} \sqrt{\alpha_{2}}}{\alpha_{0}} \ddot{y}
\end{aligned}
$$

Modelo de Facchinett et al.

$$
\begin{aligned}
\ddot{y}+2 \zeta_{w} \delta_{w}+\delta_{w}^{2} y & =\frac{C_{L 0}}{2 \pi^{3} S t^{2}\left(m^{*}+1\right)} v-\frac{\gamma}{\mu} \dot{y} \\
\ddot{v}+\epsilon\left(v^{2}-1\right) \dot{v}+v & =A \ddot{y}
\end{aligned}
$$




\section{Resultados de Simulação}

O software desenvolvido para a simulação dos modelos incorporou as equações no formato original, como apresentado pelos respectivos autores em seus trabalhos originais. A simulação do modelos de Hartlen\&Currie em formato normalizado, equações (3) e (4), é dificultada pela presença de um termo integral que causa um offset no valor médio das séries temporais obtidas para as variáveis de estado. Os valores dos parâmetros de calibração dos modelos também foram utilizados sem modificação.

As figuras 3,4 e 5 mostram os gráficos resultantes da simulação dos modelos originais. A tabela 2 mostra os valores de $m^{*}$ e de $\zeta$ usados nas simulações assim como os símbolos usados nos gráficos. São apresentados gráficos de: amplitude do oscilador fluido, v; amplitude de vibração, $y$; fase relativa entre a força e o deslocamento; fase relativa entre a força e a aceleração; força resultante no cilindro; e coeficiente de massa adicional.

A figura 6 mostra gráficos resultantes da simulação das vibrações de um cilindro forçadas através de uma fonte ideal. O sistema dinâmico resultante é linear e o gráfico da figura $6 \mathrm{~b}$ mostra o coeficiente de massa adicional resultante. Os gráficos de massa adicional resultantes dos modelos simulados, figuras $3 \mathrm{~h}, 4 \mathrm{~h}$ e $5 \mathrm{~h}$, mostram grande semelhança com o caso linear, apresentando apenas pequenas distorções na faixa de sincronização. Pode-se perceber que é possível estabelecer a faixa de sincronização prevista pelos modelos através de sua curva de massa adicional.

A figura 3 mostra os resultados da simulação do modelo de Hartlen\&Currie. Para $m^{*} \zeta<0.33$, ver figura $3 \mathrm{~b}$, os resultados da simulação do modelo apresentam valores de amplitude reduzida de vibração bem discrepantes dos dados experimentais. Segundo a simulação, para $m^{*} \zeta=0.033$ tem-se $y \gg 1$.

A leitura do artigo original de Hartlen\&Currie [7], no qual o modelo foi proposto, mostra que a calibração de seus parâmetros de ajuste foi feita com base nos dados resultantes dos experimentos de Feng. Estes experimentos, realizados em túneis de vento, possuem $m^{*} \approx 100$ e resultam em valores altos de $m^{*} \zeta$. Em particular, o valor $m^{*} \zeta=0.251$, foi utilizado por Govardhan\&Williamson [6] para reproduzir um experimento equivalente aos de

Tabela 1: Comparação dos Modelos Analisados

\begin{tabular}{rccc} 
Padrão & Hartlen\&Currie & Iwan\&Blevins & Facchinetti et al. \\
\hline$\Delta_{m_{a}}$ & $-\frac{1}{m^{*}+1}$ & $-\frac{1}{m_{4}^{*}+1}$ & 0 \\
$\Delta_{\zeta_{a}}$ & 0 & $\frac{\alpha^{2}}{2 \pi^{2} S t\left(m^{*}+1\right) \delta_{w}}$ & $\frac{\gamma}{2 \delta_{w} \mu}$ \\
$\epsilon$ & $\alpha$ & $2 \alpha_{1}$ & $\epsilon$ \\
$\mu$ & $\frac{3 \gamma}{\alpha}$ & 4 & 1 \\
$s(v)$ & $\frac{1}{2 \pi^{3} S t^{2}\left(m^{*}+1\right)} \int_{t_{0}}^{t} v d t$ & $\frac{1}{2 \pi^{3} S t^{2}\left(m^{*}+1\right)} \frac{\alpha_{4}}{\sqrt{\alpha_{2}}} v$ & $\frac{C_{L 0}}{2 \pi^{3} S t^{2}\left(m^{*}+1\right)} v$ \\
$f(y)$ & $b \delta_{n} \ddot{y}$ & $\frac{2}{\pi} \frac{\alpha_{4} \sqrt{\alpha_{2}}}{\alpha_{0}} \ddot{y}$ & $A \ddot{y}$
\end{tabular}

Tabela 2: Legenda dos Gráficos

\begin{tabular}{cccc} 
Símbolo & $m^{*} \zeta$ & $m^{*}$ & $\zeta$ \\
\hline & & & \\
$\star$ & 0.33 & 10 & 0.033 \\
$\circ$ & 0.033 & 10 & 0.0033 \\
$\bullet$ & 0.0033 & 3.3 & 0.001
\end{tabular}


Feng. Esta discrepância mostra que o modelo, como proposto originalmente, não é possui universalidade, não podendo, portanto, ser utilizado sem um processo de recalibração.

O gráfico de fase relativa, figura $3 \mathrm{c}$, apresentam saltos entre os valores $-\pi$ e $\pi$ que são causados pelo método de cálculo. Na representação polar de números complexos, os $\operatorname{argumentos} \pi \mathrm{e}-\pi$ são necessariamente iguais e representam pontos na semi-reta negativa de números reais.

A figura 4 apresenta os resultados da simulação numérica do modelo de Iwan\&Blevins, reescrito por Parra\&Aranha em Relatório Técnico da Escola Politécnica [9].

A amplitude de vibração prevista pelas simulação é compatível com os dados experimentais disponíveis. Os gráficos de fase, figuras 4c e 4d, apresentam uma resposta típica de um sistema linear forçado.

O modelo de de Langre et. al., ver figura 5, não é capaz de representar bem o pico de amplitude do fenômeno de VIV em toda a faixa de valore de $m^{*} \zeta$ testados. O valor máximo de amplitude reduzida encontrado nas simulações foi 0.2 .

Os gráficos de frequência, representados nas figuras $5 \mathrm{e}$ e $5 \mathrm{f}$, mostram claramente a ocorrência do fenômeno de sincronização ou "lock-in". Para todos os valores de $m^{*} \zeta$ testados, a frequência de oscilação prevista pelo modelo deixa de obedecer a relação de Strouhal mesmo depois da faixa de sincronização.

Todos os modelos, em maior ou menor grau, são capazes de reproduzir uma faixa de sincronização em torno da velocidade reduzida de ressonância da estrutura, ou seja, $U_{r}=5$. Todos os modelos apresentaram um alargamento da faixa de sincronização conforme se diminui o valor de $m * \zeta$.

Para $m^{*} \zeta=0.0033$ o modelo de Iwan\&Blevins apresentou valores de frequência de vibração efetiva que deixam de respeitar a relação de Strouhal mesmo depois da faixa de sincronização. Com relação a este aspecto, o modelo de de Langre et al. apresentou comportamento similar ao de Iwan\&Blevins para todas os valores de $m^{*} \zeta$ simulados.

A relação de Govardhan\&Williamson para frequência de vibração efetiva, figura 1d não foi obtida como resultado de nenhum dos modelos simulados.

Nenhum modelo apresentou amplitude reduzida de vibração dividido em ramo inicial, ramo superior e ramo inferior. Em particular nenhum modelo foi capaz de estender as vibrações para velocidade reduzidas próximas de $U_{r}=10$.

O modelo que se destaca na análise quantitativa de seus resultados é o modelo de Iwan\&Blevins. Uma característica marcante deste modelo é sua capacidade de prever o pico de resposta com base nos parâmetros $m^{*} \zeta$.

Estudos sobre modificações dos modelos de Hartlen\&Currie e de Iwan\&Blevins foram realizados. No primeiro foi feita a incorporação de um termo de "stall", termo da forma $\Delta_{\zeta_{a}}$ no modelo padrão querepresenta um amortecimento que varia em função de $U_{r}$. Como não houve processo de calibração posterior, os valores de pico obtidos não são condizentes com dados experimentais. Os gráficos da figura 7 apresentam os resultados obtidos da simulação. Pode-se notar, figura 7a, que as amplitudes de vibração se mantiveram próximas o que significa uma melhora com relação ao modelo original. Com a modificação, a curva de massa adicional para o valor de $m^{*} \zeta=0.0033$ deixou de apresentar a assíntota em $C_{a}=-1$ o que é uma inconsistência. A presente modificação do modelo de Iwan\&Blevins foi feita visando uma alteração na curva de massa adicional. Para tanto, foi feita uma variação do valor de $m^{*}$. A frequência natural do oscilador estrutural foi variada consistentemente. No oscilador fluido, a frequência natural foi variada com o objetivo de recuperar a curva de Govardhan\&Williamson, ver figura 1d. Essas modificações são equivalentes à inclusão de termos que são proporcionais à aceleração dos osciladores. Os resultados obtidos pela simulação do modelo modificado podem ser observadas na figura 8. Em sua tese de doutorado, Fujarra [5] propôs uma modificação no modelo de Iwan\&Blevins que consistiu na inclusão de um coeficiente de massa adicional e na alteração do coeficiente de sustentação, ambos em função da velocidade reduzida. Os resultados obtidos na presente modificação do modelo de Iwan\&Blevins são semelhantes aos resultados obtidos por Fujarra através no caso em que foi incorporada apenas sua modificação relativa à massa adicional. 


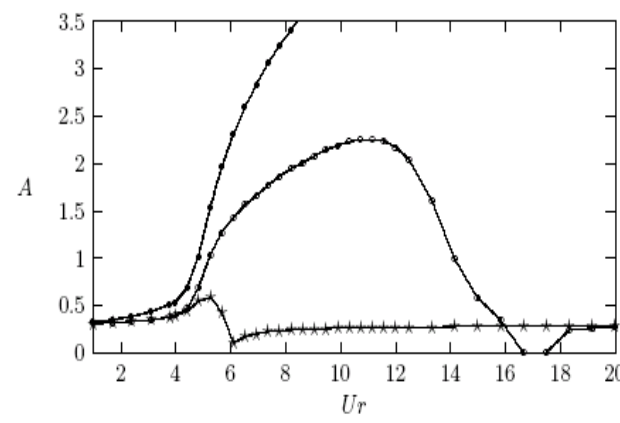

(a) Amplitude da variável de estado, $v$

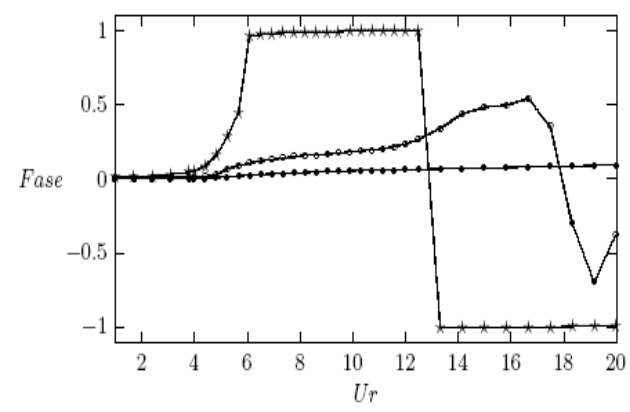

(c) Fase relativa entre a força de sustentação e o deslocamento do cilindro

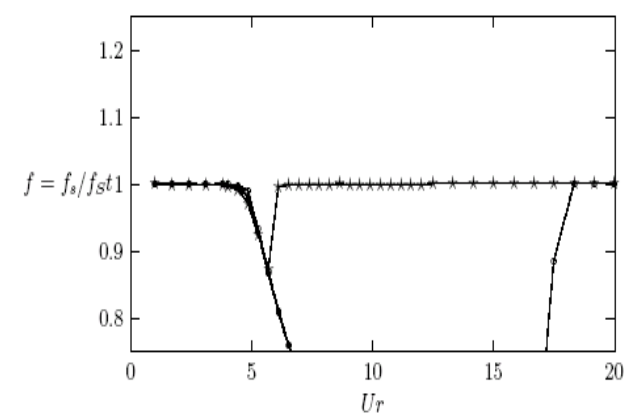

(e) Frequência efetiva de vibração, normalizada por $f_{s t}$

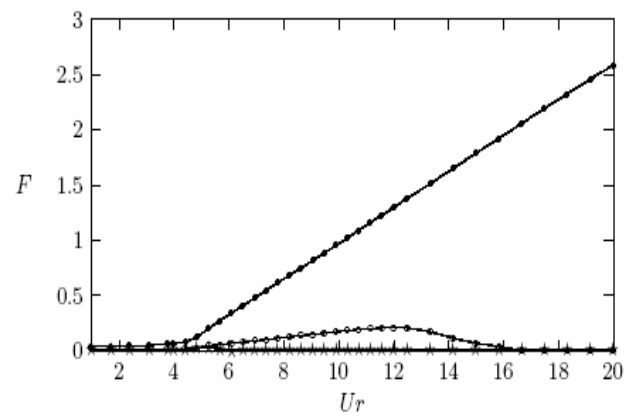

(g) Força de sustentação resultante

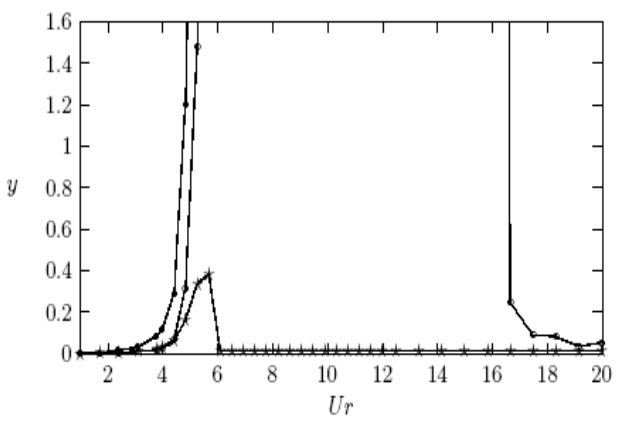

(b) Amplitude de vibração do cilindro, $y$

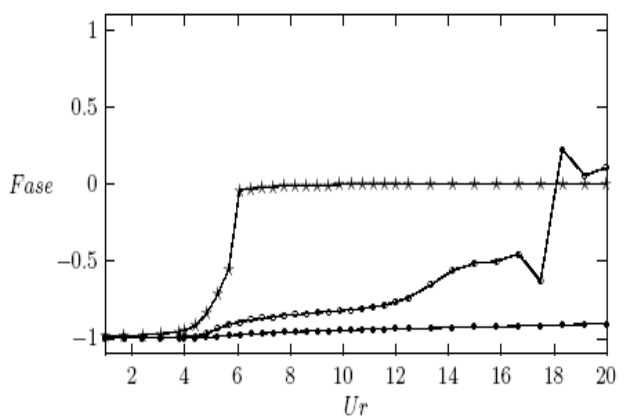

(d) Fase relativa entre a força de sustentação e a aceleração do cilindro

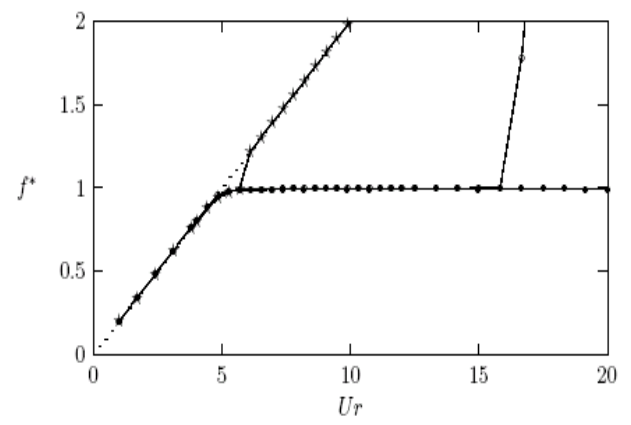

(f) Frequência efetiva de vibração, normalizada por $f_{n}$

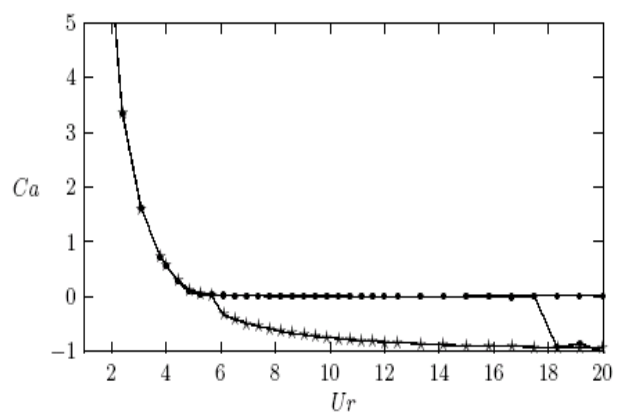

(h) Coeficiente de massa adicional

Figura 3: Resultados obtidos da simulação do modelo de Hartlen\&Currie original 


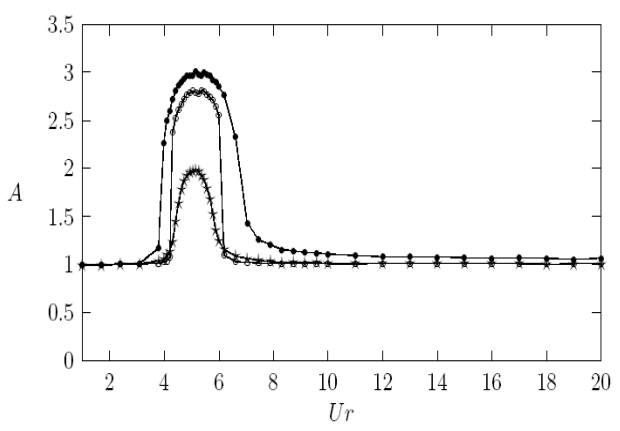

(a) Amplitude da variável de estado, $v$

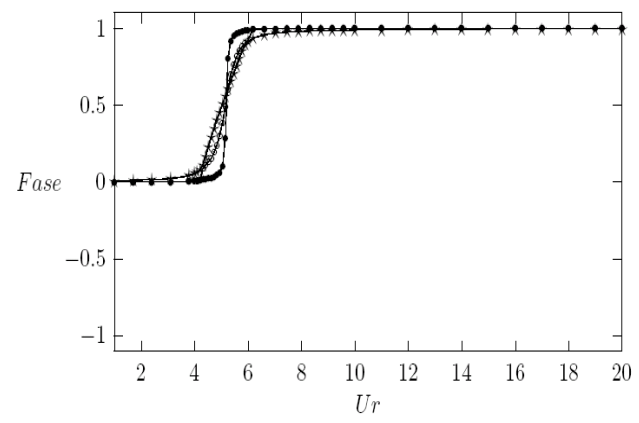

(c) Fase relativa entre a força de sustentação e o deslocamento do cilindro

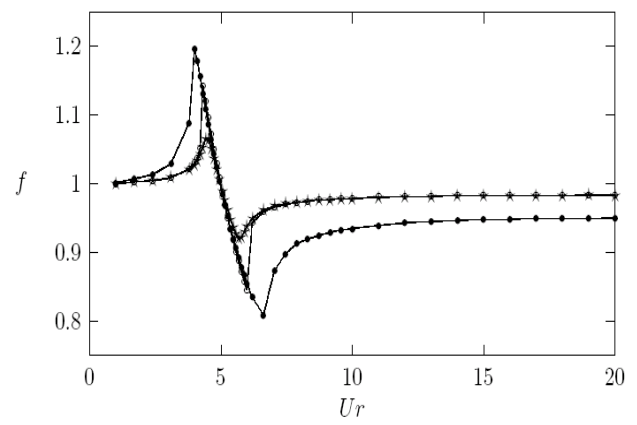

(e) Frequência efetiva de vibração, normalizada por $f_{s t}$

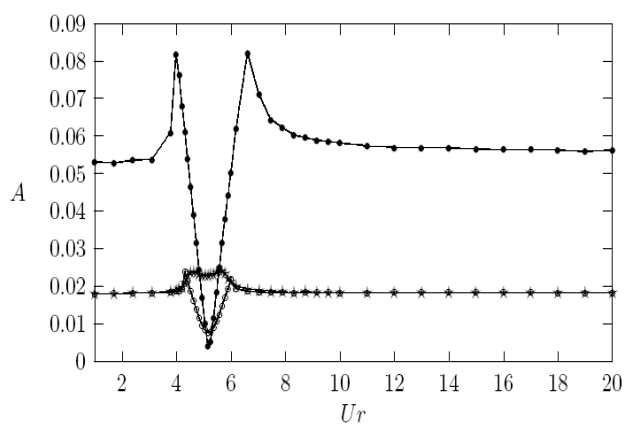

(g) Força de sustentação resultante

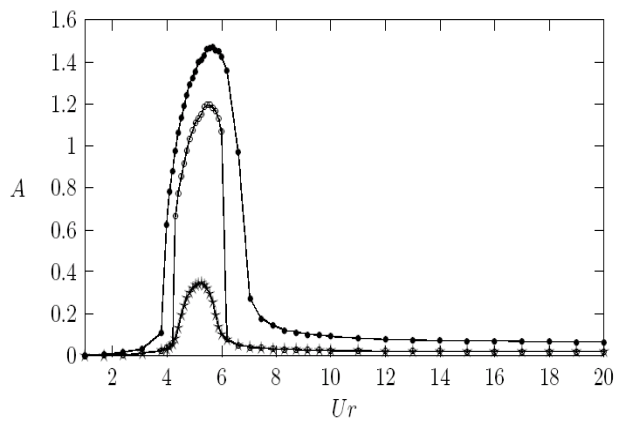

(b) Amplitude de vibração do cilindro, $y$

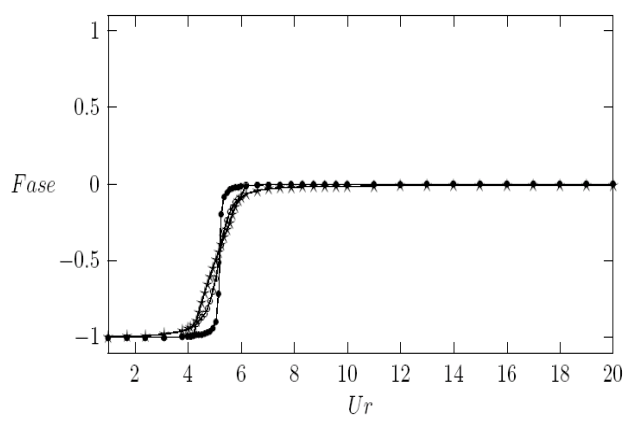

(d) Fase relativa entre a força de sustentação e a aceleração do cilindro

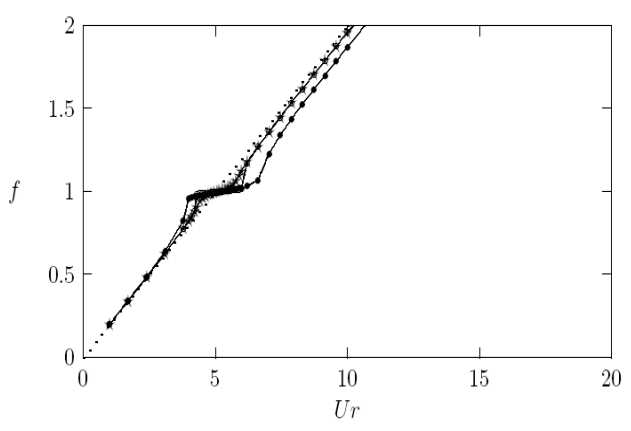

(f) Frequência efetiva de vibração, normalizada por $f_{n}$

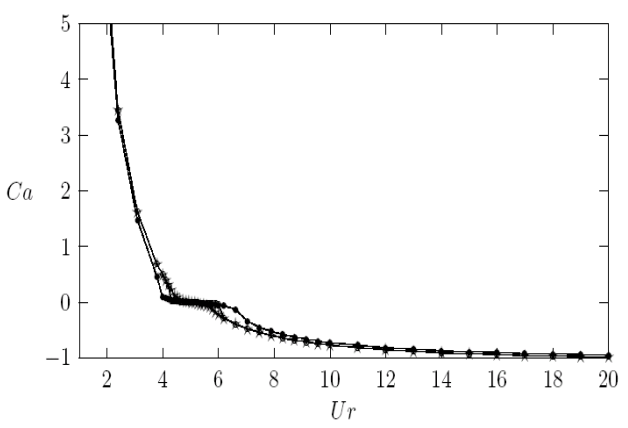

(h) Coeficiente de massa adicional

Figura 4: Resultados obtidos da simulação do modelo de Iwan\&Blevins original 


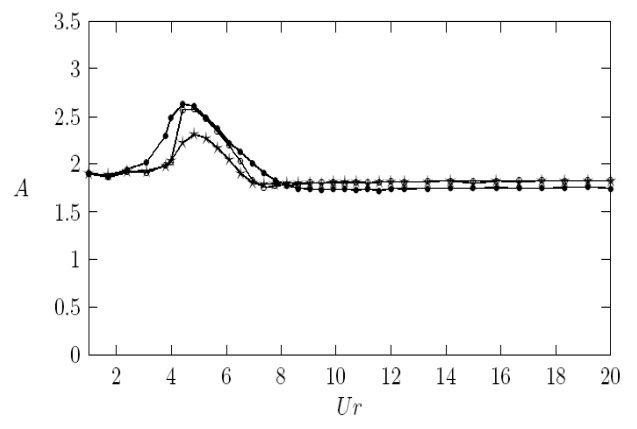

(a) Amplitude da variável de estado, $v$

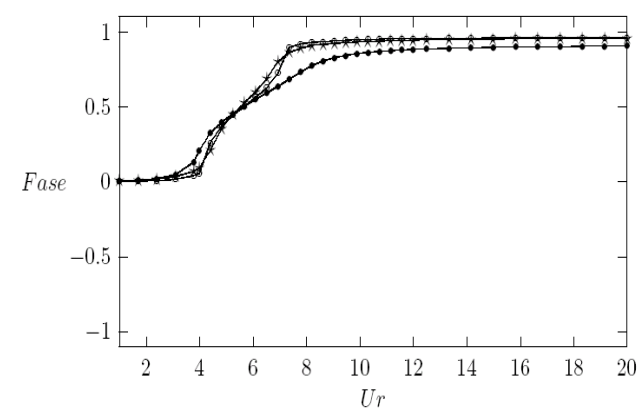

(c) Fase relativa entre a força de sustentação e o deslocamento do cilindro

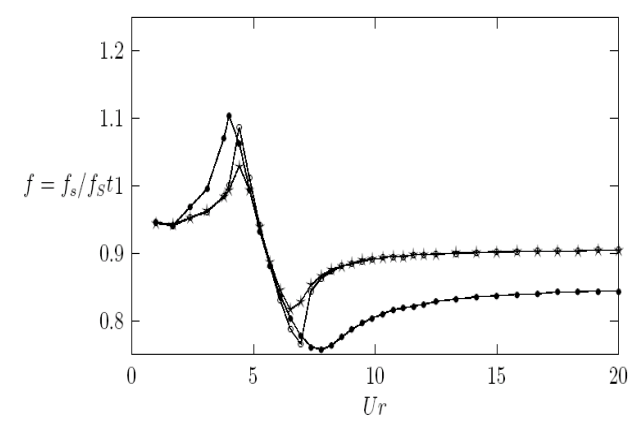

(e) Frequência efetiva de vibração, normalizada por $f_{s t}$

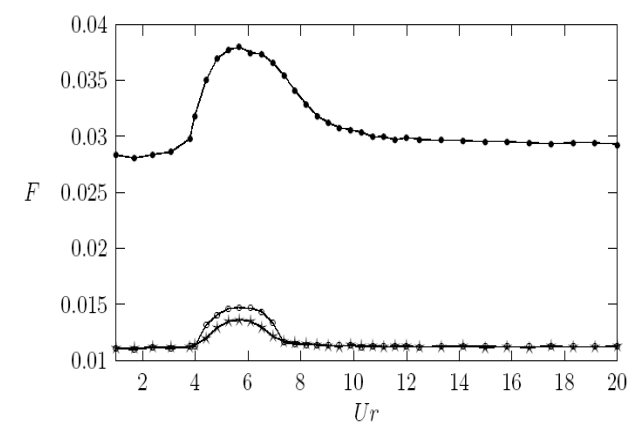

(g) Força de sustentação resultante

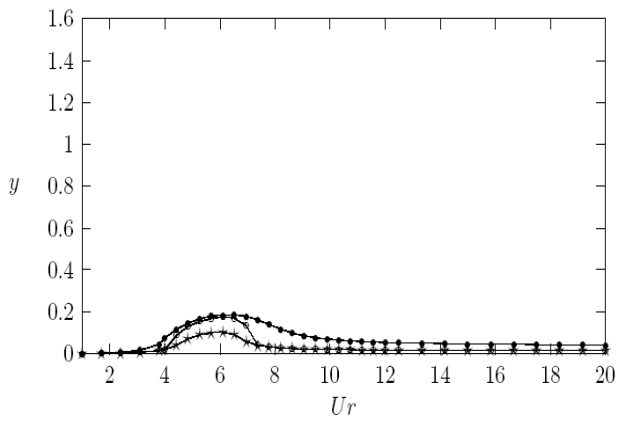

(b) Amplitude de vibração do cilindro, $y$

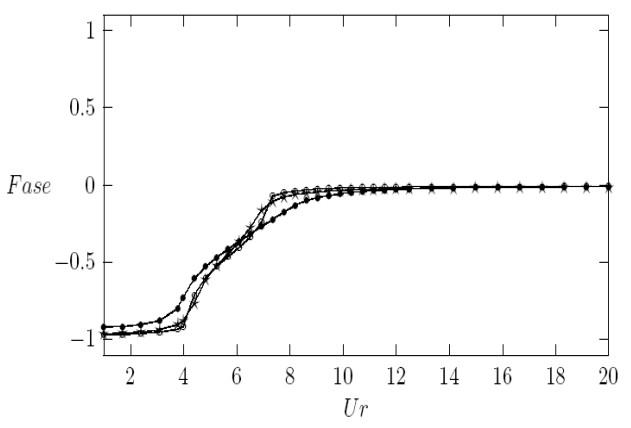

(d) Fase relativa entre a força de sustentação e a aceleração do cilindro

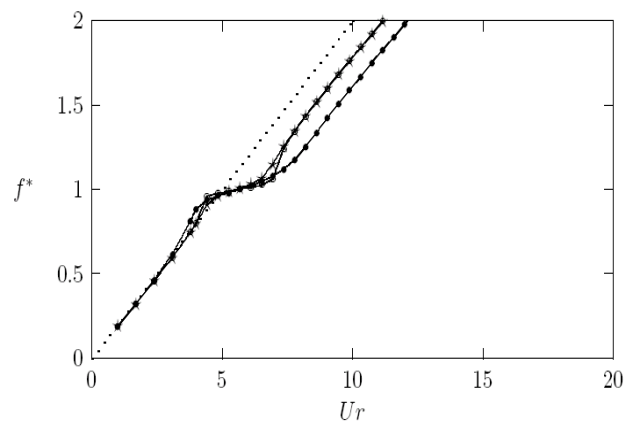

(f) Frequência efetiva de vibração, normalizada por $f_{n}$

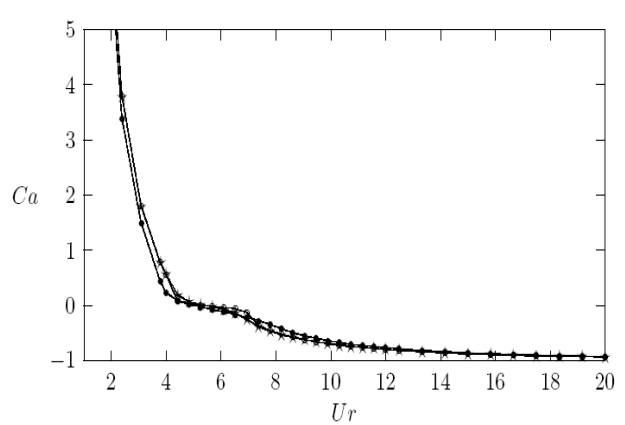

(h) Coeficiente de massa adicional

Figura 5: Resultados obtidos da simulação do modelo de Facchinetti\&de Langre\&Biolley 


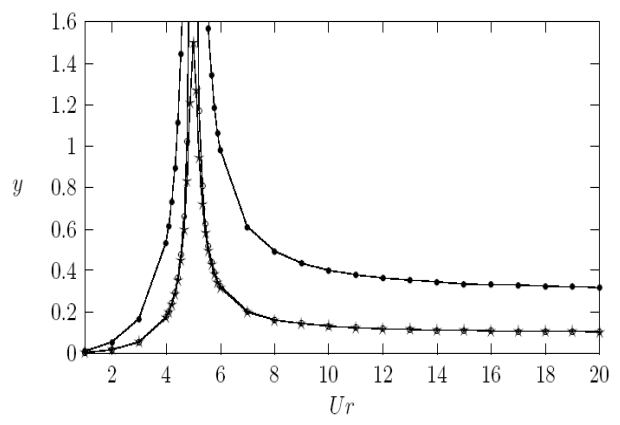

(a) Amplitude de vibração do cilindro, $y$

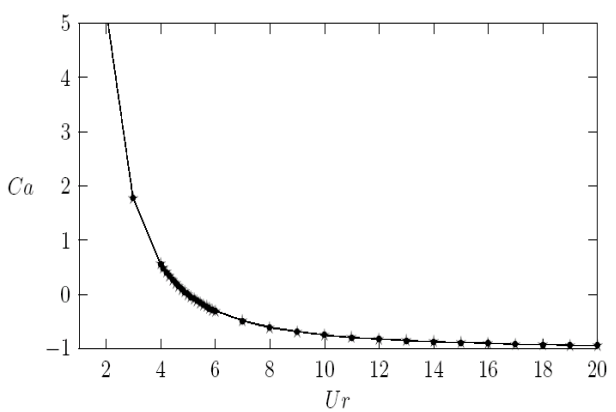

(b) Coeficiente de Massa adicional

Figura 6: Vibrações Forçadas por Fonte Ideal

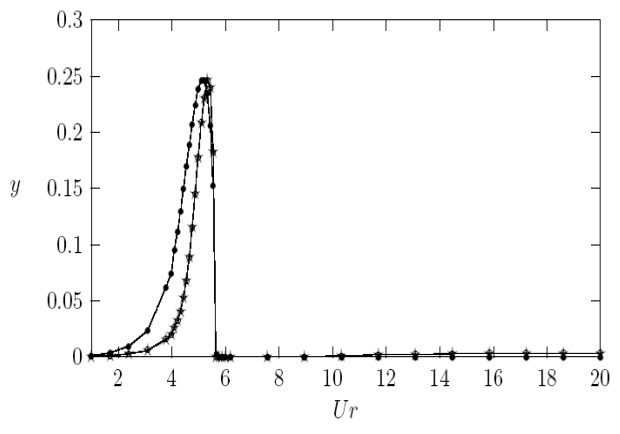

(a) Amplitude de vibração do cilindro, $y$

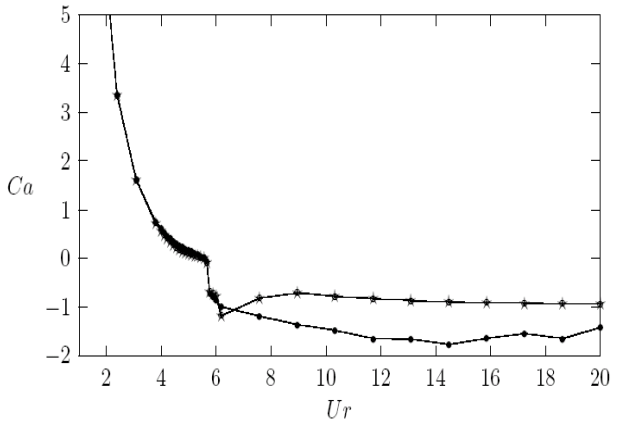

(b) Coeficiente de massa adicional

Figura 7: Resultados de Simulação obtidos através do modelo de Hartlen\&Currie modificado.

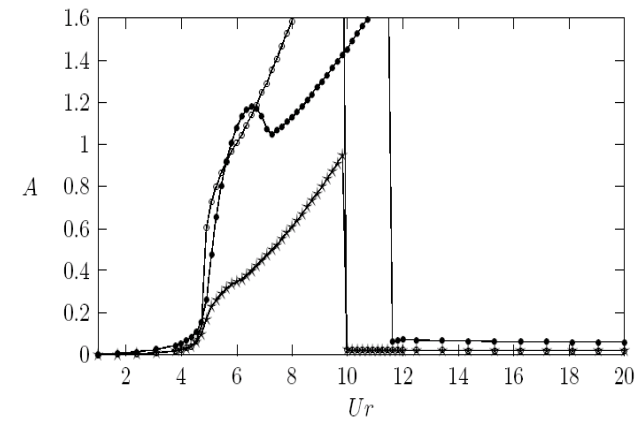

(a) Amplitude de vibração do cilindro, $y$

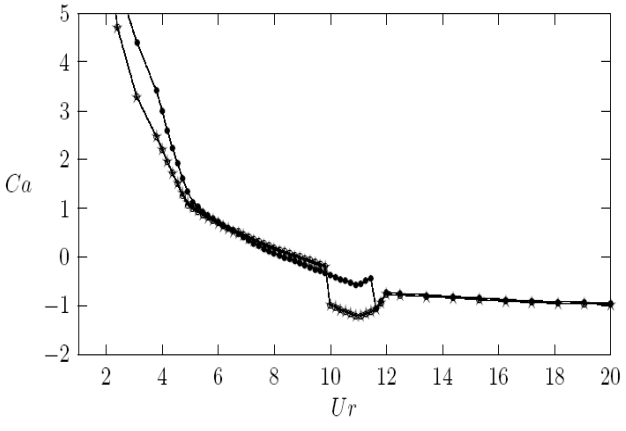

(b) Coeficiente de massa adicional

Figura 8: Resultados de Simulação obtidos através do modelo de Iwan\&Blevins modificado.

\section{Conclusão}

O modelo de Hartlen\&Currie, como originalmente proposto, é adequado para valores de $m^{*} \zeta$ típicos de ar. Não por acaso, este modelo foi calibrado com base em dados de experimentos feitos por Feng [4] apud. [7] em túneis de vento. A sua utilização na prática 
deve ser vista com reserva. Estudos de modificações foram feitos sobre este modelo. Os resultados da incorporação de um termo de "stall" ao modelo permitiram controlar os valores de pico da amplitude de vibração.

O modelo de de Langre et al. subestima a amplitude de vibração o que causa, portanto, restrições a seu uso na prática. Com relação ao coeficiente de massa adicional, bastante comentado no artigo original, sua reprodução a partir da simulação não é exclusividade deste modelo, pelo contrário.

O modelo de Iwan\&Blevins apresenta uma boa capacidade de reproduzir a amplitude de pico para diferentes valores de $m^{*} \zeta$. Esta característica já era esperada uma vez que há comentários relacionados na literatura [9]. Com relação ao ramo inferior de amplitude, não ocorre comportamento similar, ou seja, este ramo não é previsto nem mesmo qualitativamente. Os estudos de modificações do modelo de Iwan\&Blevins através da incorporação de um termo de força de acoplamento proporcional à diferença de acelerações dos dois osciladores mostrou resultados diferentes do que vinha sendo observado para os outros modelos.

A despeito das discrepâncias entre os modelos estudados e, entre os modelos e os dados experimentais, os resultados obtidos das simulações para o coeficiente de massa adicional, $C_{a}$, são qualitativamente corretos em todos os modelos. Tendo como referência o valor de $C_{a}$ calculado da simulação de um sistema linear forçado, verificou-se que a menos da faixa de sincronização, quando $C_{a} \approx 0$, os três modelos estudados apresentaram curvas semelhantes com a mesma assíntota para $U_{r} \rightarrow \infty$. Esta assíntota é a mesma conseguida no caso linear.

Em outras palavras, os resultados obtidos para os valores de $C_{a}$ não podem ser considerados uma medida de qualidade para modelos fenomenológicos no que diz respeito à sua capacidade de predição da dinâmica do sistema de VIV. Esta "robustez" do coeficiente de massa adicional decorre unicamente da consistência interna dos diversos modelos.

Os estudos sobre modificações do modelo de Iwan\&Blevins chegaram a gerar curvas de $C_{a}$ quantitativamente melhores, inclusive recuperando o zero da curva em valores de $U_{r}$ mais próximos dos valores experimentais disponíveis.

As considerações de Govardhan\&Williamson sobre a transição da fase entre a força de sustentação e o deslocamento do cilindro permitem estimar a velocidade reduzida na qual o coeficiente de massa adicional será nulo como sendo a velocidade reduzida em que ocorre a transição entre os ramos superior e inferior de amplitude [6]. No entanto, a comparação destes valores com os dados experimentais de Vikestad et al. [12] e Fujarra [5] não corrobora a teoria de Govardhan\&Williamson.

A comparação dos resultados das simulações com dados experimentais conclui que é bastante limitada a aplicabilidade dos modelos fenomenológicos [2]. Apenas o modelo de Iwan\&Blevins foi capaz de reproduzir a amplitude de pico constatada experimentalmente.

Com relação ao desenvolvimento futuro, a mais promissora é a abordagem de Aranha [1] através da equação de Ginzburg-Landau. Esta mesma abordagem fundamenta os modelos fenomenológicos permitindo reavivar o interesse sobre esse enfoque.

Os estudos realizados indicam que futuras modificações devem incluir dados experimentais a respeito das bifurcações relacionadas aos padrões de emissão de vórtices tendo em vista prever adequadamente a transição entre os ramos de resposta.

Os modelos fenomenológicos podem, ainda, ser expandidos através da incorporação de outro núcleo de vibração representando a vibração longitudinal.

\section{REFERÊNCIAS}

[1] Aranha, J.A.P.: Weak Three Dimensionality of a Flow Around a Slender Cylinder: The GinzburgLandau Equation, A ser publicado (2004)

[2] Cunha, L.D. Vibração Induzida por Vórtices: Análise Crítica de Modelos Fenomenológicos, Dissertação de Mestrado da EPUSP (2005)

[3] Facchinetti, M.L.; de Langre, E.; Biolley, F.: Coupling of Structure and Wake Oscillators in VortexInduced Vibrations, Journal of Fluids and Structures, 19 (2004)

[4] Feng, C.C.: The Measurements of Vortex-Induced effects in Flow Past Stationary and Oscillating Circular and D-Section Cylinders, Msc. Thesis (unpublished), (1968) 
[5] Fujarra, A.L.C.: Estudos Experimentais e Analíticos das Vibrações Induzidas pela Emissão de Vórtices em Cilindros Rígidos e Flexíveis, Tese de Doutorado da EPUSP, (2002)

[6] Govardhan, R.; Williamson, C.H.K.: Modes of Vortex Formation and Frequency Response os a Freely Vibrating Cylinder, Journal of Fluids Mechanics, Volume 420, (2000)

[7] Hartlen, R.T.; Currie, I.G.: Lift Oscillator Model of Vortex-Induced Vibration, Proceedings of the ASCE, (1970)

[8] Iwan, W.D.: The Vortex Induced oscillation of Elastic Structural Elements, Transactions of the ASME, (1975)

[9] Parra, P.;Aranha, J.A.P.: Vibrações Induzidas por Emissão de Vórtices: Modelos Fenomenológicos e Experimentos, Relatório Técnico da EPUSP (1996)

[10] Sarpkaya: Vortex Induced Vibrations, Journal of Fluids and Structures, Volume 9, Issue 1 (1979)

[11] Sarpkaya: Critical Review, Special document (2003)

[12] Vikestad, K.; Vandiver, J.K.; Larsen, C.M.: Added Mass and Oscilation Frequency for a Circular Cylinder Subjeted to Vortex-Induced Vibrations and External Disturbance, Journal of Fluids and Structures (2000)

[13] Williamson, C.H.K.; Govardhan, R.: Critical Mass in Vortex-Induced Vibration of a Cylinder, European Journal Mechanics B/Fluids, Volume 23, (2004)

[14] Willianson, C.H.K.; Govardhan, R.: Vortex-Induced Vibrations, Annual Reviews in Fluid Mechanics, (2004) 